\title{
DNA Hypomethylation and Histone Variant macroH2A1 Synergistically Attenuate Chemotherapy-Induced Senescence to Promote Hepatocellular Carcinoma Progression
}

\author{
Michela Borghesan ${ }^{1,2}$, Caterina Fusilli ${ }^{3}$, Francesca Rappa ${ }^{4}$, Concetta Panebianco ${ }^{5}$, \\ Giovanni Rizzo', Jude A. Oben', Gianluigi Mazzoccoli², Chris Faulkes ${ }^{6}$, Illar Pata \\ Antonella Agodi ${ }^{8}$, Farhad Rezaee ${ }^{9}$, Shane Minogue', Alessandra Warren ${ }^{1,10}$, \\ Abigail Peterson", John M. Sedivy", Julien Douet ${ }^{12,13}$, Marcus Buschbeck ${ }^{12,13}$, \\ Francesco Cappello ${ }^{4,14}$, Tommaso Mazza ${ }^{3}$, Valerio Pazienza ${ }^{5}$, and Manlio Vinciguerra ${ }^{1,5,14,15}$
}

\begin{abstract}
Aging is a major risk factor for progression of liver diseases to hepatocellular carcinoma (HCC). Cellular senescence contributes to age-related tissue dysfunction, but the epigenetic basis underlying drug-induced senescence remains unclear. macroH2A1, a variant of histone $\mathrm{H} 2 \mathrm{~A}$, is a marker of senescence-associated heterochromatic foci that synergizes with DNA methylation to silence tumor-suppressor genes in human fibroblasts. In this study, we investigated the relationship between macroH2A1 splice variants, macroH2A1.1 and macroH2A1.2, and liver carcinogenesis. We found that protein levels of both macroH2A1 isoforms were increased in the livers of very elderly rodents and humans, and were robust immunohistochemical markers of human cirrhosis and HCC. In response to the chemotherapeutic and DNA-demethylating agent 5-aza-deoxycytidine (5-aza-dC), transgenic expression of macroH2A1 isoforms in HCC cell lines prevented the emergence of a senescent-
\end{abstract}

like phenotype and induced synergistic global DNA hypomethylation. Conversely, macroH2A1 depletion amplified the antiproliferative effects of 5-aza-dC in HCC cells, but failed to enhance senescence. Senescence-associated secretory phenotype and whole-transcriptome analyses implicated the p38 MAPK/IL8 pathway in mediating macroH2A1-dependent escape of HCC cells from chemotherapy-induced senescence. Furthermore, chromatin immunoprecipitation sequencing revealed that this hepatic antisenescence state also required active transcription that could not be attributed to genomic occupancy of these histones. Collectively, our findings reveal a new mechanism by which drug-induced senescence is epigenetically regulated by macroH2A1 and DNA methylation and suggest macroH2A1 as a novel biomarker of hepatic senescence that could potentially predict prognosis and disease progression. Cancer Res; 76(3); 1-13. (92016 AACR.

\section{Introduction}

Hepatocellular carcinoma (HCC) is the sixth most frequently diagnosed cancer and the second leading cause of cancer-related death worldwide. Prognosis is poor, as only $10 \%$ to $20 \%$ of patients with HCC are eligible for surgery; without surgery, the expected survival is less than 6 months (1). Aging is the major risk factor for HCC, which is often triggered by the metabolic syndrome and nonalcoholic fatty liver disease (NAFLD), along with the insurgence of cirrhosis (2). In agreement with the inflammaging theory, in which aging accrues inflammation, old age seems to favor the progression of liver diseases toward HCC (2). In the absence of disease, the liver possesses a unique regenerative
"Institute for Liver and Digestive Health, Royal Free Hospital, Uni-
versity College London, London, United Kingdom. ${ }^{2}$ Division of Inter-
nal Medicine, Department of Medical Sciences, IRCCS "Casa Sollievo
della Sofferenza" Hospital, San Giovanni Rotondo, Italy. ${ }^{3}$ Bioinfor-
matics Unit, IRCCS "Casa Sollievo della Sofferenza"-Mendel Labo-
ratory, Rome, Italy. ${ }^{4}$ Department of Experimental Biomedicine and
Clinical Neurosciences, Section of Human Anatomy, University of
Palermo, Palermo, Italy. ${ }^{5}$ Gastroenterology Unit, IRCCS "Casa Sol-
lievo della Sofferenza" Hospital, San Giovanni Rotondo, Italy. ${ }^{6}$ School
of Biological and Chemical Sciences, Queen Mary University of
London, London, United Kingdom. "Department of Gene Technolo-
gy, Tallinn University of Technology (TTU), IVEX Lab, Tallinn, Estonia.
${ }^{8}$ Department GF Ingrassia, University of Catania, Catania, Italy.
9Department of Cell Biology, University Medical Center Groningen,
Groningen, the Netherlands. ${ }^{10}$ Centre for Education and Research on
Aging (CERA) and the ANZAC Research Institute, Concord RG
Hospital, University of Sydney, Sydney, Australia. "Department of
Molecular Biology, Cell Biology and Biochemistry, Brown University,
Providence, Rhode Island. ${ }^{12}$ Institute for Predictive and Personalized
Medicine of Cancer, Campus Can Ruti, Badalona, Spain. ${ }^{13}$ Josep Carreras Institute for Leukaemia Research, Campus ICO-HGTP, Campus Can Ruti, Badalona, Spain. ${ }^{14}$ Euro-Mediterranean Institute of Science and Technology (IEMEST), Palermo, Italy. ${ }^{15}$ School of Science and Technology, Nottingham Trent University, Nottingham, United Kingdom.

Note: Supplementary data for this article are available at Cancer Research Online (http://cancerres.aacrjournals.org/).

V. Pazienza and M. Vinciguerra contributed equally to this article.

Corresponding Author: Manlio Vinciguerra, University College London, Rowland Hill Street, London NW3 2PF, UK. Phone: 0044-77-2548-8381; Fax: 004420-7433-2852; E-mail: m.vinciguerra@ucl.ac.uk

doi: 10.1158/0008-5472.CAN-15-1336

(ㅇ)2016 American Association for Cancer Research. 
capacity and preserved functional performance during aging (2). Nevertheless, the aging process increases the risk of hepatic functional and structural impairment and metabolic risk. In this respect, mouse models of metabolic syndrome with NAFLD reveal features of accelerated aging, impaired regeneration, and an increased incidence of $\operatorname{HCC}(2,3)$. Interventions to clear senescent hepatocytes in a neoplastic-prone tissue microenvironment delay the emergence of HCC in rats (4). Cellular senescence can thus regulate tumor suppression, as well as contribute to age-related loss of tissue function through the secretion of multiple proinflammatory molecules, such as IL6 and IL8, as part of a senescence-associated secretory phenotype (SASP; ref. 5). Moreover, changes in nuclear chromatin structure that occur during senescence are highlighted by the formation of punctate and visible DNA foci in $4^{\prime}, 6$-diamidino-2-phenylindole (DAPI)-stained senescent cells, known as senescence-associated heterochromatin foci (SAHF; ref. 5). In vertebrates, histone variants provide continuous regulation of nucleosome turnover across the entire lifespan of the organism, in addition to being expressed in terminally differentiated cells, and they are a chief cellular strategy to regulate transcription and cellular metabolism. Specifically, the histone $\mathrm{H} 2 \mathrm{~A}$ variant macroH2A1 is enriched in SAHF of senescent human fibroblasts (5), but its mechanistic role in cellular senescence is poorly understood. macroH2A1 is composed of a domain $66 \%$ homolog to histone $\mathrm{H} 2 \mathrm{~A}$, and it stands out because of its unique structure, whereby a C-terminal linker connects the histone fold domain to a macro domain. This domain protrudes from the compact structure of the nucleosome, likely affecting the function and organization of the surrounding chromatin, and is conserved in multiple functionally unrelated proteins throughout the animal kingdom. macroH2A1 exists as two alternatively exon-spliced isoforms, macroH2A1.1 and macroH2A1.2, which bind in vitro with different affinities to O-acetyl-ADP-ribose (OAADPR), a small metabolite produced by the protein/histone deacetylase SIRT1 (6). Historically, macroH2A1 has been implicated in X chromosome inactivation and transcriptional repression: this view has recently been challenged by reports that have linked macroH2A proteins to signal-induced gene activation (7-9). macroH2A1 isoforms have taken center stage in the plasticity of stem cell differentiation and in the pathogenesis of many cancers, providing an exciting, yet poorly understood, link to metabolism and nutrients $(10,11)$.

In the liver, genome binding and transcriptomic studies using knock-out (KO) mice have shown that macroH2A1 participates in the pathogenesis of NAFLD and fat-induced obesity (12-18). Moreover, increased expression of macroH2A1 isoforms in the liver might be used as a diagnostic and prognostic marker for HCC (3). In the aging murine and primate livers, we observed an increased age-associated localization of macroH2A1 to regions of pericentromeric heterochromatin (19). It is, however, unknown whether increased expression of macroH2A1 during aging could favor the onset of HCC, which is characterized by profound epigenetic alterations, such as promoter-specific hypermethylation and global DNA hypomethylation (20). Hypomethylating agent 5-aza-deoxycytidine (5-aza-dC, decitabine) and its derivatives inhibit DNA methyltransferases proteins and are chiefly used for the treatment of nonsolid malignancies; however, they also exert antitumor effects in HCC cells by inducing senescence (21-24). In human fibroblasts, macroH2A1 deficiency combined with 5-aza-dC treatment activates tumor-suppressor genes and inhibits cell proliferation (25).
The aim of this study is to investigate the interplay between macroH2A1 isoforms and DNA methylation during "healthy" liver aging and hepatic carcinogenesis, using aged and $\mathrm{KO}$ rodent models, macroH2A1-isoform specific transgenic HCC cell lines, and human liver tissues.

A model emerged where macroH2A1 marks but does not affect physiologic hepatic senescence. In the presence of HCC-associated or chemotherapy-induced DNA hypomethylation, high levels of macroH2A1 might instead favor HCC progression through a distinct p38 MAPK/IL8-dependent mechanism.

\section{Materials and Methods}

\section{Animals}

All mice in C57/BL6 background were housed in a clean, temperature-controlled mouse facility on a 12-hour light/dark cycle on a standard diet. Liver tissues from young (3 months) and old (30 months) animals were provided by the European Molecular Biology Laboratory, Monterotondo, Italy. Harvesting procedures were approved by the local Ethical Committee (Monterotondo, Italy) and were in accordance with national and European regulations. macroH2A1 KO mice were kindly provided by Professor John Pehrson (University of Pennsylvania, Philadelphia, PA; ref. 26). Aged macroH2A1 KO mice were produced and housed at Brown University in a specific pathogen-free Association for Assessment and Accreditation of Laboratory Animal Care-certified barrier facility. Heterozygous animals were bred, and macroH $2 \mathrm{~A} 1+/+$ and $-/-$ littermates were retained for further analysis. All procedures were approved by the Brown University Institutional Animal Care and Use Committee. Animals were euthanized using isofluorane anesthesia, followed by cervical dislocation. Liver tissue was rapidly removed, flash-frozen in liquid nitrogen or embedded in optimal cutting temperature compound, and stored at $-80^{\circ} \mathrm{C}$.

\section{Human biopsies and histology}

Liver specimens were obtained from patients undergoing clinically indicated hepatic biopsies (27), with appropriate institutional ethical approval and patient consent and in accordance with the Code of Ethics of the World Medical Association (Declaration of Helsinki). Liver paraffin-embedded sections (4- $\mu \mathrm{m}$ thick, 10 per condition) were stained with hematoxylin \& eosin (H\&E) and Masson's trichrome for histologic evaluation, as described previously $(3,28)$. Diagnostic classification of NAFLD and fibrosis/cirrhosis was performed using a semiquantitative scoring system that grouped histologic features into broad categories (steatosis, hepatocellular injury, portal inflammation, fibrosis, and miscellaneous features; ref. 29). Human healthy liver samples from four old (72-81 years old) and four young (33-48 years old) healthy subjects were obtained from OriGene.

\section{Immunohistochemistry and X-gal staining}

Sections from human liver specimens were processed by Masson trichrome staining for histologic evaluation. Immunostaining on human biopsies and murine livers was performed using the iVIEW DAB Detection Kit for the Ventana BenchMark XT-automated slide stainer. Primary antibodies for macroH2A1.1 and macroH2A1.2 were previously generated (3). Anti-5-Methylcytosine (33D3) antibody was obtained from Active Motif (cat. no. 39649); anti-GLB1/Beta-Galactosidase Antibody (clone 5H2) IHC-plus LS-B10217 was obtained from LSBio. macroH2A1.1, 
macroH2A1.2, and anti-GLB1 primary antibodies were diluted 1:100; anti-5-Methylcytosine primary antibody was diluted $1: 1,000$. Positive and negative controls were run concurrently. Means of triplicate counts were used for statistical analyses. For XGal staining of murine livers, frozen sections of $5-\mu \mathrm{m}$ thickness were used and processed as previously described (30). Slides were counterstained with eosin, mounted with aqueous medium, and observed with an optical microscope (Nikon ECLIPSE Ni) connected to a digital camera (DS-Fi2). The percentage of blue staining, indicating $\beta$-galactosidase activity, was calculated in 10 random high-power field (HPF) at $400 \times$ magnification and expressed as means. Also, after performing quantitative analysis, the results were expressed in a semiquantitative scale $(-: 0 \% ;+$ : 1\%-33\%; ++: 34\%-66\%; +++: 67\%-100\%). All analyses were performed in triplicate by three independent pathologists.

Constructs, cell cultures, and proliferation/migration assays

Stable differential expression of macroH2A1.1 or macroH2A1.2 isoforms tagged with GFP in HepG2 and in Huh-7 cells was achieved by lentiviral transduction. cDNAs encoding macroH2A1 isoforms were placed under the control of a constitutive EF1 $\alpha$ promoter in the HIV1-based self-inactivating lentiviral vector, modified to allow expression of macroH2A isoforms as $\mathrm{N}$-terminal fusions with a monomeric fluorescent protein mTagGFP. Viral preparations were produced by transient transfection in HEK293T cells, and concentrated 100-fold by low-speed centrifugation $(6,000 \times g$ for 16 hours). HepG2 and Huh-7 cells were transduced with virus at multiplicity of infection $>10$ for 24 hours in the presence of $4 \mu \mathrm{g} / \mathrm{mL}$ Polybrene. Stably transduced cell populations were established and used in subsequent experiments without clonal selection. To generate appropriate controls, cells were also stably transduced with the empty vector (expressing only mTagGFP) and maintained in similar culture conditions to macroH2A1-expressing cells. For knock-down experiments, HepG2 were transduced with retroviral macroH2A-specific shRNA cassettes (31). Transient transfection of macroH2A1 isoforms in mouse HCC Hepa1-6 cells was carried out as described (17). HepG2 and Huh-7 cells were treated for 72 hours with 12 $\mu \mathrm{mol} / \mathrm{L}$ of 5-aza-dC (decitabine; Sigma). For p38 MAPK activity inhibition and IL8 receptor blockade, cells were concomitantly treated with $10 \mu \mathrm{mol} / \mathrm{L}$ SB202190 or $10 \mu \mathrm{mol} / \mathrm{L}$ SB265610 (Sig$\mathrm{ma})$, respectively. Cell migration and cell proliferation were assessed by wound-healing assay and by MTT assay respectively as previously described $(17,32)$.

\section{SASP analyses}

sICAM, IL1 $\beta$, IL6, and IL8 levels were assessed in HepG2 and Huh-7 cell supernatants, upon treatment with 5-aza-dC and or p38 MAPK/IL8 pathway chemical inhibitors, using a customized human MILLIPLEX MAP (multi-analyte panels) Luminex system (Merck Millipore; ref. 12), according to the manufacturer's instructions.

\section{$\boldsymbol{\beta}$-galactosidase assay}

The Senescence $\beta$-Galactosidase Staining Kit (Cell Signaling Technology; \#9860) was used in HepG2 and in Huh-7 cells to evaluate $\beta$-galactosidase activity at $\mathrm{pH} 6$, a known characteristic of senescence, according to the manufacturer's instructions.

\section{DNA methylation assay (LUMA)}

LUMA was used to quantify genome-wide methylation levels in cell lines and mouse liver (33). Genomic DNA was extracted using a commercial kit (Qiagen). LUMA measures the percentage of global methylation using the ratio of DNA cleavage by methylation-sensitive (HpaII) and methylation-insensitive (MspI) restriction enzymes, followed by polymerase extension assay by pyrosequencing to determine cleavage. The LUMA method was validated using DNA controls of known human DNA methylation status. Genome-wide methylation was expressed as a percentage as previously described (33).

\section{Gene expression}

Total RNA was isolated from livers of mice or cells using TRIzol (Invitrogen) and quantified using NanoDrop 1000 spectrophotometer (Thermo Scientific). After RNA quality verification, 1 to 2 $\mu \mathrm{g}$ was used to prepare cDNA. In Hepa1-6 HCC cells, Cellular Senescence RT $^{2}$ Profiler PCR Array profiles were assessed following the manufacturer protocol (SABioscience). Quantitative PCR was performed using SYBR Green (SIGMA) in an ABI-PRISM qPCR cycler (Applied Biosystems), as described previously (34). Glyceraldehyde-3-phosphate dehydrogenase (GAPDH) transcript was used as internal control for normalization using the 2- $\Delta \Delta \mathrm{CT}$ method. Primer sequences were as follows: GAPDH, Forward CATGGCCTTCCGTGTTCCTA, Reverse GCGGCACGTCAGATCCA; GLB1, Forward TTTACGTGGGCAACTTCTC, Reverse TACTTGACCCTTGGACCAC.

\section{Western blot}

Chromatin and nuclei protein fractions from macroH2A1.1GFP, macroH2A1.2-GFP, and GFP transgenic HepG2 and Huh-7 cells were isolated (35). Cell pellets were suspended in buffer A $\left(10 \mathrm{mmol} / \mathrm{L} \mathrm{HEPES}, 10 \mathrm{mmol} / \mathrm{L} \mathrm{KCl}, 1.5 \mathrm{mmol} / \mathrm{L} \mathrm{MgCl}_{2}, 340\right.$ $\mathrm{mmol} / \mathrm{L}$ sucrose, $10 \%$ glycerol, $1 \mathrm{mmol} / \mathrm{L}$ DTT), incubated on ice, and centrifuged at $1,300 \times g$ at $4^{\circ} \mathrm{C}$ for 5 minutes to separate supernatant from nuclei. Nuclei fractions were washed once and lysed for 30 minutes in buffer B ( $3 \mathrm{mmol} / \mathrm{L}$ EDTA, $0.2 \mathrm{mmol} / \mathrm{L}$ EGTA, $1 \mathrm{mmol} / \mathrm{L}$ DTT) and centrifuged at $1,700 \times g$ at $4^{\circ} \mathrm{C}$ for 5 minutes to separate the supernatant (nuclei) from the pellet (chromatin). Cytoplasmic and nuclear protein extraction from rodent liver parenchyma and hepatoma cells, and immunoblotting analyses were performed as described previously (3). The histone fraction was enriched using acid extraction and 2,2,2trichloroacetic acid precipitation. Primary antibodies were obtained from Activ Motif (macroH2A1.1 and macroH2A1.2), Cell Signaling Technology [phospho-p38 MAPK (Thr180/ Tyr182), p38 MAPK, phospho-MSK1 (Thr581), MSK1, phospho-ATF2 (Thr71), and ATF2], and Abcam (GFP, PCNA). Antibodies against histone H3 (Activ Motif), GAPDH (Cell Signaling Technology), and $\beta$-actin (Santa Cruz Biotechnology) were used to normalize protein levels.

\section{Chromatin immunoprecipitation sequencing and RNA- sequencing}

For both chromatin immunoprecipitation sequencing (ChIPSeq) and RNA-sequencing (RNA-Seq), Genomix4life S.r.l. performed sample quality control by next-generation sequencing. ChIP was performed with a modified protocol as described previously (36). Chromatin complexes were lysed, extracted, digested with micrococcal nuclease (MNase), immunoprecipitated (using $4 \mu \mathrm{g}$ of anti-GFP antibody, ab290; Abcam), and reverse crosslinked. DNA fragments (200 to 1,000 bp) were then cleaned up and eluted using the components of the Pierce Agarose ChIP Kit, ChIP-Grade Protein A/G Plus Agarose and 
Proteinase K (Pierce). Primer sequences for positive controls of macroH2A1 isoform binding to chromatin in HepG2 cells were LAMA5_-2.8K_F: AGAATGCCCCCACCCTAC, LAMA5_-2.8K_R: TCCCAAGGTCAGGAGTTCAC; PTPRN_-199_F: TGTAAAGCACCTTGGAGACTG, PTPRN_-39_R: CAGAGAGCACAGCAGGAGAG. For RNA-Seq, total RNA was extracted from GFP-, macroH2A1.1-GFP, or macroH2A1.2-GFP transgenic HepG2 cell lines with TRIzol Reagent (Invitrogen). Indexed libraries were prepared from $2 \mu \mathrm{g} /$ ea purified RNA with the TruSeq Total Stranded RNA Sample Prep Kit (Illumina) according to the manufacturer's instructions. Libraries were quantified using the Agilent 2100 Bioanalyzer (Agilent Technologies) and pooled so that each index-tagged sample was present in equimolar amounts; the final concentration of the pooled samples was $2 \mathrm{nmol} / \mathrm{L}$. Pooled samples were then subjected to cluster generation and sequencing using an Illumina HiSeq 2500 System (Illumina) in a $2 \times 100$ paired-end format at a final concentration of $8 \mathrm{pmol} / \mathrm{L}$.

\section{Bioinformatics analyses}

RNA-Seq and ChIP-Seq raw data (.fastq files) underwent quality control analysis using the FastQC software package. Short reads were discarded, trimmed, or adapter-cleaned where necessary. Transcripts were assembled by aligning around 30 million reads per sample toward the hg19 reference genome by Bowtie2. Uniquely mapped reads that fell within each feature of interest were counted and RPKM normalized. Twenty million reads for each ChIP-seq experiment were mapped against hg19 by Bowtie. A peak calling strategy implemented in the Partek Genomics Suite, which was set to tolerate broadly spreading and weaker patterns, was used to predict the regions of the genome where the ChiPed histone was bound. Differentially expressed and differentially bound genes were subjected to in-silico functional enrichment analysis by QIAGEN's Ingenuity Pathway Analysis. Functions and pathways were considered significantly over-represented if $P$ values were $<0.05$. Genes of relevant pathways were marked with colors to represent their expression directions.

\section{Statistical analyses}

The RNA-Seq study was designed with a sample size of 14 and conferred a power (1-beta) of 0.8 , when the significance threshold (alpha) was set at 0.05 . We tolerated less than $5 \%$ of false positives and considered only genes with at least a 1.5 -fold difference (FDR-corrected $P$ value $<0.05$ ). Differential expression between genes was determined by two-way ANOVA in the Partek Genomics Suite, and any random batch effects were minimized using the Batch Effect Removal tool of Partek. One-tailed binomial tests were used to calculate $P$ values, which compared each sample with their respective reference (GFP) in the ChIP-Seq experiment. A low $P$ value meant there were significantly more reads in the sample than in the reference sample. The scaled change in intensity of each signal was compared with the reference sample. The change was scaled by a ratio of the number of reads for each sample on a per-chromosome basis. A scaled change $>1$ indicated more enrichment in the sample than in the reference. The whole procedure of functional enrichment analysis was based on prior calculation of the activation $z$-scores, which infer the activation states of predicted transcriptional regulators, functions, and pathways. To confer solidity to the analysis, we considered $z$-scores $>2$ (minimum activation threshold) and $<-2$ (minimum inhibition threshold).

Results were expressed as mean \pm SE. Comparisons between means were made by appropriate Student $t$ tests. Differences of proportions were assessed by one-tailed $\chi^{2}$ tests. Differences were considered as significant when $P<0.05$.

\section{Results}

Increased levels of macroH2A1 isoforms in old livers and in human HCC are associated with $\boldsymbol{\beta}$-galactosidase expression

Murine hepatic expression of macroH2A1.1 and macroH2A1.2 increases from newborn to adult age (26). We detected significantly increased expression of macroH2A1 isoforms $(P<0.01$ for macroH2A1.1 and $P<0.001$ for macroH2A1.2, respectively) in the liver of old (30 months) compared with young ( 3 months) mice (Fig. 1A). Strikingly, in healthy humans, the proportion of nuclei that were immunopositive for macroH2A1 isoforms was significantly higher in the livers of old versus young individuals (macroH2A1.1: $45.2 \% \pm 2.4 \%$ in old vs. $36.5 \% \pm 1.5 \%$ in young, respectively, $P<0.05$; macroH2A1.2: $56.2 \% \pm 2.9 \%$ in old vs. $36.4 \% \pm 2.4 \%$ in young, respectively, $P<0.05$; Fig. $1 \mathrm{~B}$ and $\mathrm{C}$ ). Hepatocyte nuclei from older subjects were larger than in younger subjects (Fig. 1B and C), most likely reflecting liver cell polyploi$\mathrm{dy}$, which indicates terminal differentiation and senescence. Consistent with previous studies (3), hepatocyte immunopositivity for macroH2A1.1 and macroH2A1.2 was significantly higher in viral cirrhosis and HCC compared with healthy young controls (Fig. 1B and C). A significantly higher number of cells immunopositive for $\beta$-galactosidase ( $\beta$-gal), a key marker of cellular senescence, paralleled the severity of liver disease (control < cirrhosis < HCC) in humans, as demonstrated by H\&E and Trichrome staining (Fig. 2A-D). Taken together, these findings demonstrate that hepatic expression of macroH2A1 isoforms increases with healthy aging and during liver cirrhosis and HCC, and this is paralleled by the growing expression of the senescence marker $\beta$-gal.

Global DNA hypomethylation in HCC but not in old livers

Global changes in DNA methylation may play a pleiotropic role during aging and age-related pathologies, such as cancer. To investigate this, we assessed global genomic DNA methylation in the liver using LUMA. The median LUMA genome-wide methylation was similar, approximately $70 \%$, in liver from old (30 months old) and young (3 months) mice (Fig. 3A). Moreover, we did not detect differences in immunopositivity for cytosine-5 methylation in liver samples from old versus young humans (Fig. $3 \mathrm{~B}$ and C). Global genomic DNA hypomethylation is observed along the liver disease spectrum (20). Accordingly, nuclear immunopositivity for cytosine-5 methylation was decreased in liver biopsies from HCC patients compared with cirrhotic and healthy (young and old) controls (Fig. 3B and C). Furthermore, we compared hepatic DNA methylation levels of patients with HCC and cirrhosis with those of controls in four major datasets from the Gene Expression Omnibus databank (GSE44970, GSE56588, and GSE60753) and from The Cancer Genome Atlas (TCGA; Fig. 3D). The proportion of significantly differentially methylated genes in each dataset indicated significantly higher DNA hypomethylation in HCC than in cirrhosis. Altogether, our experimental and in-silico analyses show that hepatic expression of macroH2A1 isoforms correlates with DNA hypomethylation 
A

\section{Figure 1.}

macroH2A1.1 and macroH2A1.2 expression in the whole liver of: old versus young mice (A); old and young healthy controls, patients with cirrhosis and HCC (B). Age is indicated in $A$ and $B$ ( $m$ th, months; yo, years old). A, left, representative blots of 3 to 4 animals per age group. Histone $\mathrm{H} 3$ was used as a loading control; right, quantitative measurement of macroH2A1.1 and macroH2A1.2 associated signals by densitometry. Values were normalized to $\mathrm{H} 3$ levels and expressed as a percentage of young (3 $\mathrm{mth}$ ). B, representative pictures of immunostaining performed for macroH2A1.1 and macroH2A1.2 in human liver samples from a young (33 years old) versus an old (81 years old) healthy subject

( $n=6$ per condition, 33-48 years old $\rightarrow$ young range, $72-81$ years old $\rightarrow$ old range) and in human liver samples with viral cirrhosis or HCC ( $n=10$ per condition). All nuclei of tumor cells were positive for either macroH2A1.1 or macroH2A1.2. Positivity of hepatocytes from cirrhotic livers was intermediate. C, data are expressed as mean \pm SE of 10 blindly chosen and evaluated HPF. Bar, $100 \mu \mathrm{m}$. A-C, *, $P<0.05$; **, $P<0.01$; and ***, $P<0.001$.

B

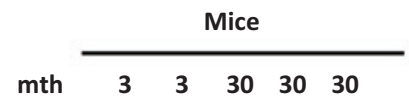

macroH2A1.1

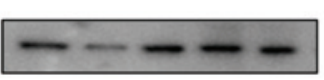

macroH2A1.2

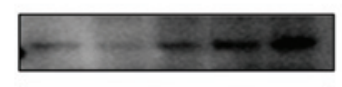

Histone H3

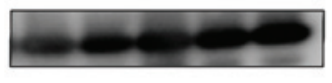

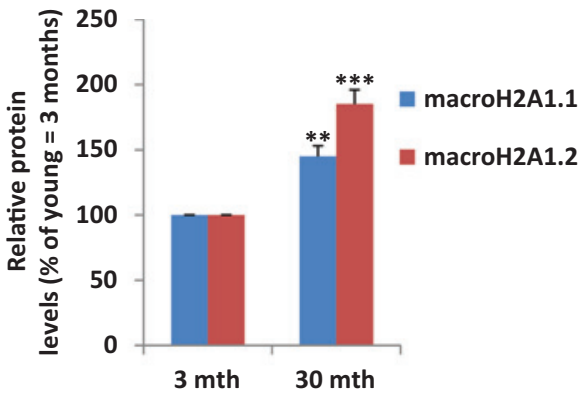

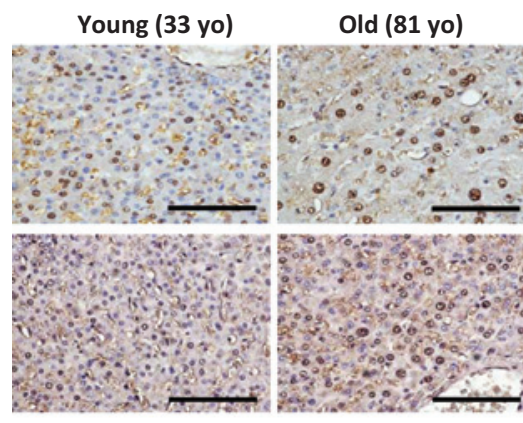

Cirrhosis
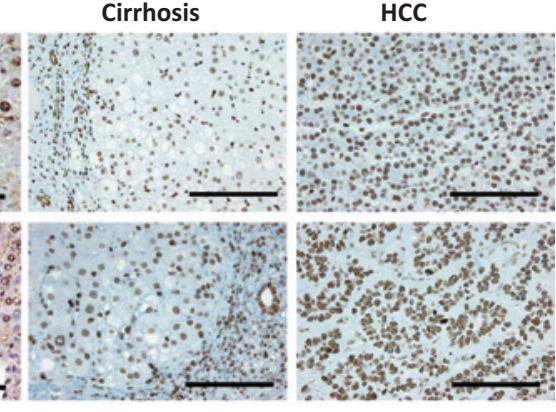

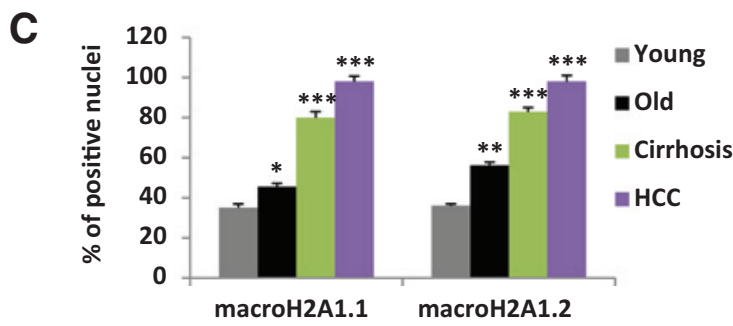

during the progression of HCC and cirrhosis, but not during healthy aging.

\section{Ablation of macroH2A1 has no effect on age-dependent} hepatocyte senescence and DNA methylation

We used whole-body KO mice for macroH2A1 (12) to investigate age-dependent hepatocyte senescence and DNA methylation. We stained the livers of 21.4- to 23.3-month-old macroH2A1 KO mice and aged-matched wild-type littermates for senescence-associated X-gal staining. There were no differences between the two genotypes (Supplementary Fig. S1A and S1B). Young, 3-month-old, mice of either genotype were negative for $\mathrm{X}$ gal staining (data not shown). Furthermore, the levels of positivity for cytosine-5 methylation in the liver of old macroH2A1 KO versus wild-type mice were comparable (Supplementary Fig. S1C and S1D). These data suggest that genetic ablation of macroH2A1 has no effect on chief markers of hepatocyte senescence and DNA methylation during physiologic aging.

macroH2A1 isoforms synergize with 5-aza-dC-induced global DNA hypomethylation and display protection against 5-azadC-dependent senescence-like phenotype in HCC cells

Promoter DNA hypermethylation and heterochromatinization are events frequently leading to tumorigenesis (37). In fibroblasts, knockdown of macroH2A1 synergizes with 5-aza-dC-induced
DNA demethylation and decreases cell proliferation (25). To investigate if macroH2A1 isoforms synergize with 5-aza-dCinduced global DNA hypomethylation and display protection against 5-aza-dC-dependent senescence, we generated lentiviralmediated stable HepG2 and Huh-7 cell lines transgenic for GFP, macroH2A1.1-GFP, or macroH2A1.2-GFP (Fig. 4). Bands at a molecular weight of approximately $70 \mathrm{KD}$, corresponding to the sum of GFP and macroH2A1 molecular weights, were detected upon nuclear/chromatin fractionation by immunoblotting, indicating a moderate increase in its expression levels (Fig. 4A and D). $\mathrm{H} 3$ served as a marker for the chromatin fraction, PCNA as a marker of both nuclear and chromatin fractions, and GAPDH as a negative marker to exclude cytoplasmic contamination. Cells transgenic for macroH2A1.1-GFP or macroH2A1.2-GFP were viable and morphologically indistinguishable from GFP transgenic cells, and 5-aza-dC did not alter the endogenous expression levels of macroH2A1.1 and macroH2A1.2 in HepG2 and Huh-7 cells (Fig. 4B and E). As expected, LUMA assay revealed an approximately $25 \%$ and $40 \%$ decrease in global DNA methylation in control GFP-expressing HepG2 and Huh-7 cells, respectively (Fig. 4C and F). Transgenic expression of either macroH2A1.1-GFP or macroH2A1.2-GFP synergized with 5aza-dC and induced greater DNA hypomethylation: of approximately $40 \%$ to $50 \%$ in HepG 2 cells, and of approximately $60 \%$ to $75 \%$ in Huh-7 cells, compared with respective untreated control 
A
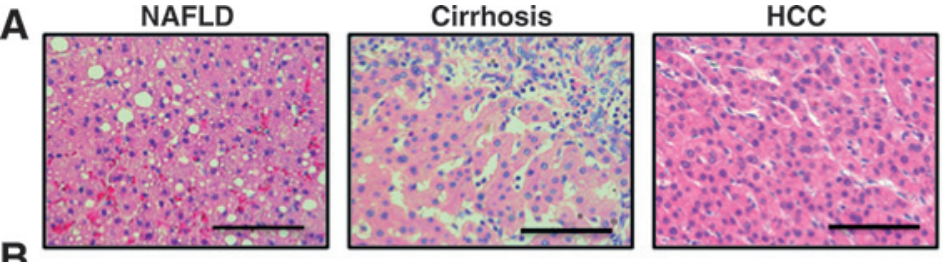

H\&E staining
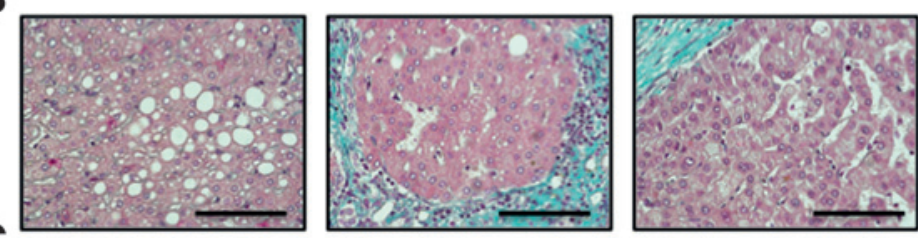

Trichrome staining

C
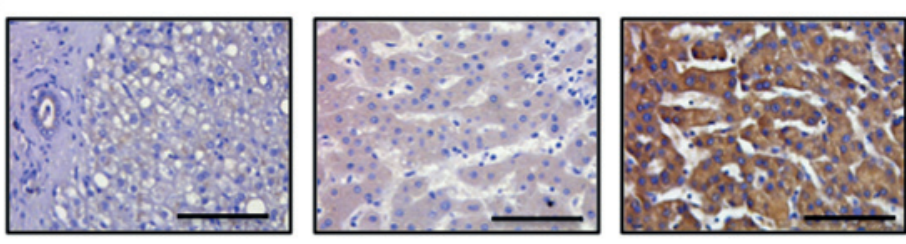

$\beta$-Gal staining

D

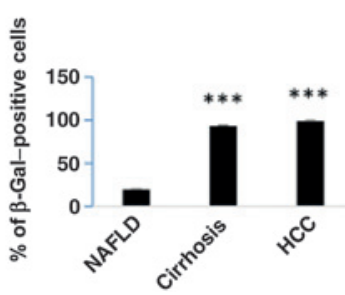

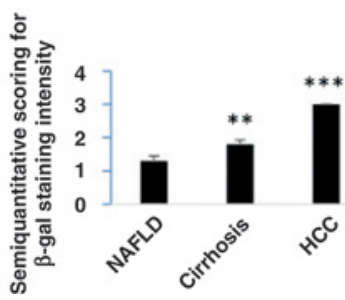

Figure 2.

$\beta$-gal expression in the liver of patients with NAFLD, cirrhosis, and HCC. A and B, histologic features of liver biopsies from patients with NAFLD, cirrhosis, and HCC. $A$, representative pictures of $\mathrm{H} \& \mathrm{E}$ staining. B, representative pictures of trichrome staining. In samples from patients with NAFLD, microvesicular and macrovesicular steatosis are apparent; in samples from patients with cirrhosis, the characteristic regenerative nodule surrounded by scattered lymphocytes and fibrous connective tissue was more visible with trichrome staining. In samples from patients with HCC, proliferation of hepatocytes and, in the top left of the trichrome image, the capsule of the tumor can be observed. $C$, representative pictures of $\beta$-gal immunostaining of human liver samples with NAFLD, cirrhosis, and $\operatorname{HCC~}(n=10$ per condition). All HCC cells were highly positive; positivity of hepatocytes with NAFLD was significantly lower and was intermediate in viral cirrhosis. Bar, 100 $\mu \mathrm{m}$. $D$, the percentage of $\beta$-gal-positive cells was calculated in 10 blindly chosen HPF at a magnification of $\times 400$ (left). Also, after performing quantitative analysis, the results were expressed in a semiquantitative scale (0, $0 \% ; 1,1 \%-33 \%$; 2, 34\%-66\%; 3, 67\%-100\%; right). Data were expressed as means $\pm \mathrm{SE}$. ${ }^{* *}, P<0.01$ and ${ }^{* * *}, P<0.001$. cells (Fig. 4C and F). HepG2 and Huh-7 cell lines treated with $12 \mu \mathrm{mol} / \mathrm{L} 5$-aza-dC for 72 hours exhibited a characteristic flattened morphology and positive staining for the senescence marker $\beta$-galactosidase (Fig. 5A and E), compared with untreated cells (Supplementary Fig. S2). Imaging-assisted quantitative analyses revealed that approximately $70 \%$ of GFP-expressing HepG2 cells, and approximately $80 \%$ of GFP-expressing Huh-7 cells, were positive for $\beta$-galactosidase staining (Fig. 5A, B, E, and F). In contrast, only approximately $40 \%$ and $35 \%$ of macroH2A1.1-GFP and macroH2A1.2-GFP HepG2 transgenic cells, and approximately $60 \%$ and $35 \%$ of macroH2A1.1-GFP and macroH2A1.2-GFP Huh-7 transgenic cells, respectively, were positive (Fig. 5A, B, E, and F), indicating an antisenescence effect of macroH2A1 isoforms. In the Hepa1-6 mouse HCC cell line, 5-aza-dC treatment induced a weaker $\beta$-gal staining compared with HepG2 and Huh-7 cells (Supplementary Fig. S3). Transient overexpression of macroH2A1.1-GFP or macroH2A1.2-GFP induced a decrease in the number of $\beta$-gal-positive Hepa1- 6 cells, accompanied by an antisenescence gene expression profile (including decreased mRNA expression of GLB1, the gene encoding for $\beta$-gal), when compared with control cells (Supplementary Fig. S3A-S3C). Senescence is characterized by a withdrawal from cell cycle and decrease in cell proliferation. In this respect, macroH2A1.1-GFP and macroH2A1.2-GFP transgene-bearing HepG2 and Huh-7 cells displayed a significant 2- to 3-fold increase in cell growth compared with control GFP-expressing cells upon 5-aza-treatment, but not in untreated cells (Fig. 5C and G). Micrograph analysis of wound closure in scratch assays showed faster cell migration in both macroH2A1.1 and macroH2A1.2 transgenic
HepG2 and Huh-7 cells upon drug treatment (Fig. 5D and H). Notably, 5-aza-dC treatment did not induce the appearance of SAHF in HCC cell lines (data not shown). In vivo, genetic ablation of macroH2A1 did not influence hepatic senescence (Supplementary Fig. S1). In vitro, knocking down the whole macroH2A1 gene in HepG2 cells (knockdown, KD) using a stable siRNA approach achieved a nearly total deletion of macroH2A1.1 and macroH2A1.2 proteins (Supplementary Fig. S4A): KD cells proliferated slower and showed a slower wound closure in the scratch assay, compared with control cells (Supplementary Fig. S4B and S4C). Mirroring in vivo data, we observed no differences in $\beta$-gal staining between control and KD cells following treatment with 5aza-dC (Supplementary Fig. S4D). Therefore, macroH2A1 isoforms facilitate the escape from the 5-aza- $\mathrm{dC}$-induced prosenescent phenotype in HCC cells.

macroH2A1 isoforms induce escape from 5-aza-dC-induced senescence through p38 MAPK/IL8 signaling in HCC cells

Tumor-suppressor proteins p53, p16, and p21 are involved in cell-cycle control and are considered master regulators of cell senescence. However, in our HCC cell models, neither 5-aza-dC nor macroH2A1 isoform transgenes altered their endogenous expression levels (data not shown). A central feature of senescence is the induction of the SASP, which implicates a complex interplay of cytokines and growth factors that are released from senescent cells (38). 5-aza-dC induces SASP in HepG2 cells (21). We thus measured the expression of a panel of SASP components in the supernatants of HepG2 and of Huh-7 cells treated with 5-aza-dC. In all cell lines, 5-aza-dC incubation increased 
Figure 3.

DNA methylation analyses in old versus young mice $(A)$; old and young healthy humans with NAFLD, cirrhosis, and HCC (B and $\mathrm{C}$ ); and methylation data mining in cirrhotic and HCC patients versus control samples (D). Age is indicated in A and $B$ (mth, months; yo, years old). A, proportion of global DNA methylation analyzed with LUMA assay is expressed as mean \pm SE of three animals per age group. $B$, representative pictures of immunostaining for 5methylcytosine in human liver samples from a young (33-year-old) versus an old (81-year-old) healthy human subject ( $n=6$ per condition, 33-48 years old $\rightarrow$ young range, 72-81 years old $\rightarrow$ old range) and in human liver samples with cirrhosis and HCC ( $n=10$ per condition). $C$, data are shown as mean \pm SE of 10 blindly chosen and evaluated HPF. Bar, $100 \mu \mathrm{m}$. ***, $P<0.001$. $D$, stacked bar plots showing the proportions of overall methylation of differentially methylated genes between patients with cirrhosis or $\mathrm{HCC}$ and healthy patients, taken from four public datasets (GSE44970, GSE56588, GSE60753, and TCGA). Red bars represent hypermethylated genes, whereas blue bars represent hypomethylated genes. Color intensity corresponds to the magnitude of differential methylation. ${ }^{* * *}, P<0.001$ between the differences in the ratios of hypomethylated genes in cirrhosis and HCC in each dataset.

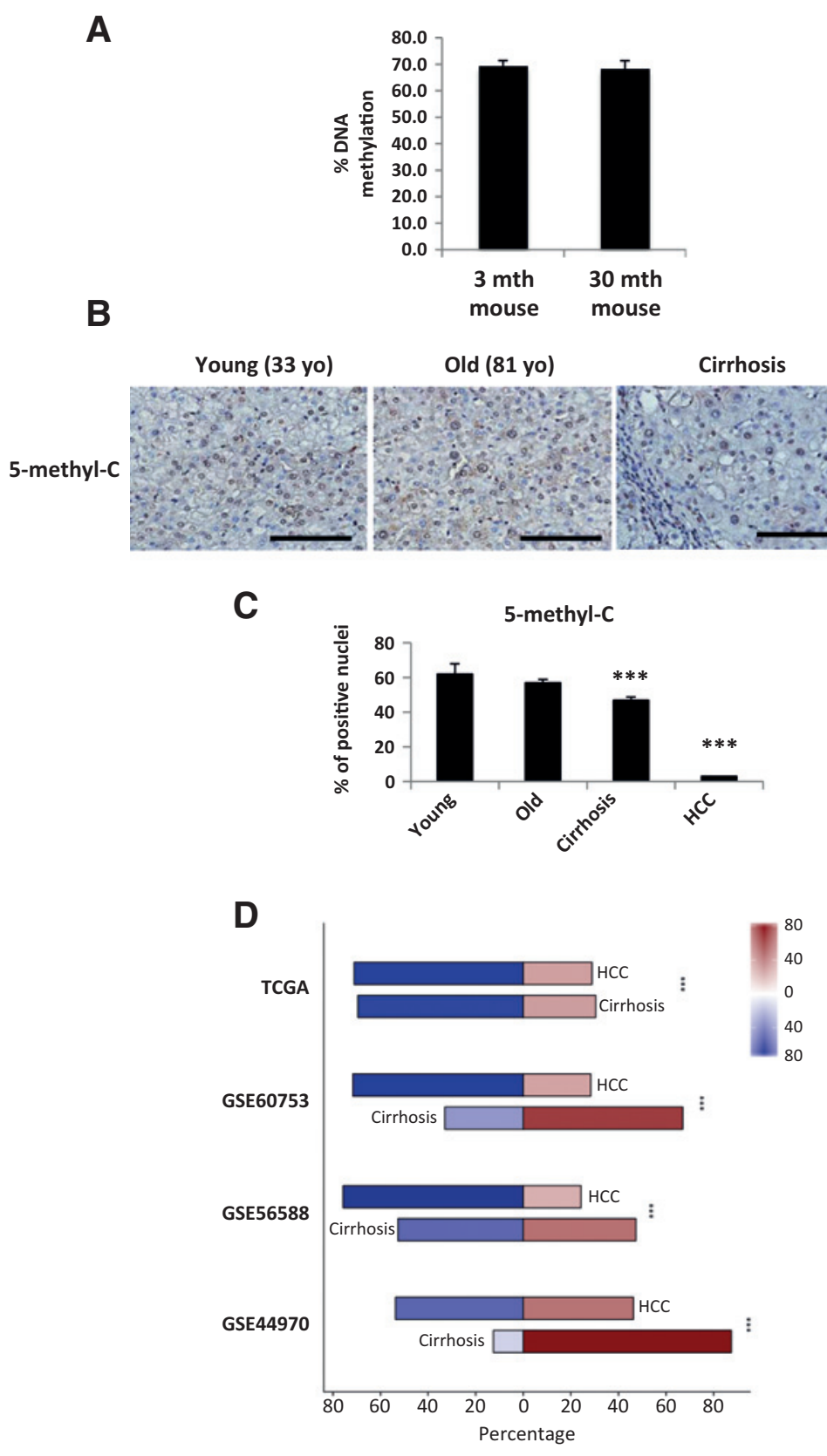

sICAM-1, IL1 $\beta$, and IL8 levels in the culture supernatants, whereas it had no effect on IL6 (Fig. 6A and Supplementary Fig. S5A). Surprisingly, in macroH2A.1- and macroH2A1.2 transgenic cells, IL8 and, to a minor extent, IL1 $\beta$ levels were significantly augmented when compared with control cells (Fig. 6A and Supplementary Fig. S5A). To gain a deeper insight into the transcriptional profiles of 5-aza-dC-treated HCC cells, we used RNA-Seq. Venn diagrams (Supplementary Fig. S6A) revealed a low overlap between differentially expressed gene in macroH2A1.1-GFP and macroH2A1.2-GFP transgenic cells versus GFP transgenic cells (Supplementary Table S1). Similarly, the transcriptional profiles of macroH2A1 KO versus GFP cells showed little overlap (Supplementary Fig. S6B). Heatmaps in Supplementary Fig. S6C and S6D represent the fold changes of the differentially expressed genes. These listed genes significantly enriched $(-\log (\mathrm{p}$-value $)>1.3)$ a number of functions and diseases, including cancer, which macroH2A1.1 and macroH2A1.2 transgenic cells over-represented (Supplementary Fig. S7A; Supplementary Table S2). Ingenuity pathway analysis (IPA) highlighted genes belonging to the p38 MAPK pathway within the pathway "cancer," which was significantly over-represented $(P<0.001)$ in macroH2A1.1-GFP and macroH2A1.2-GFP transgenic HepG2 cells (Supplementary Fig. S7B). IPA analysis suggested that $\mathrm{p} 38$ activation induced IL8 production (Supplementary Fig. S7B). To detect the activation status of p38 MAPK in HepG2 and Huh-7 cells upon 5-aza-dC treatment, we conducted an immunoblotting analysis of phospho-p38 MAPK (Thr180/ Tyr182) and downstream effectors phospho-MSK1 (Thr581) 
A

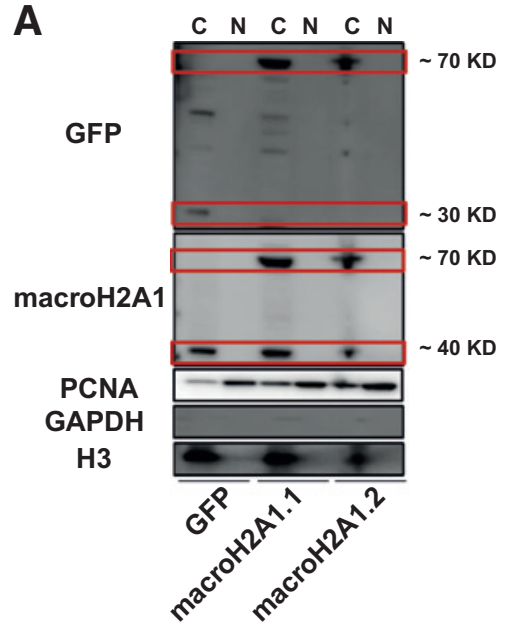

D

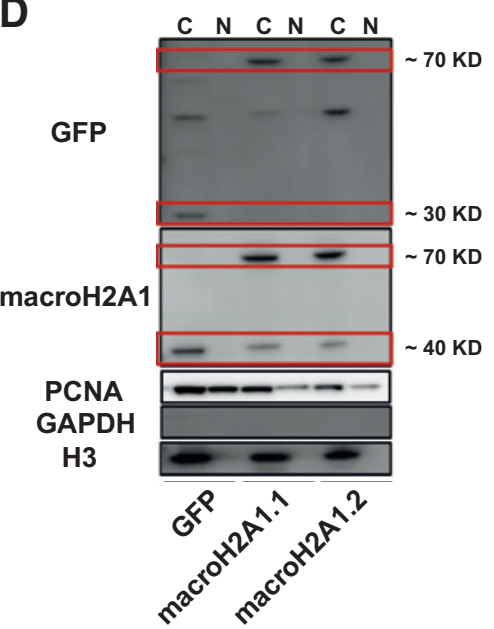

B

HepG2
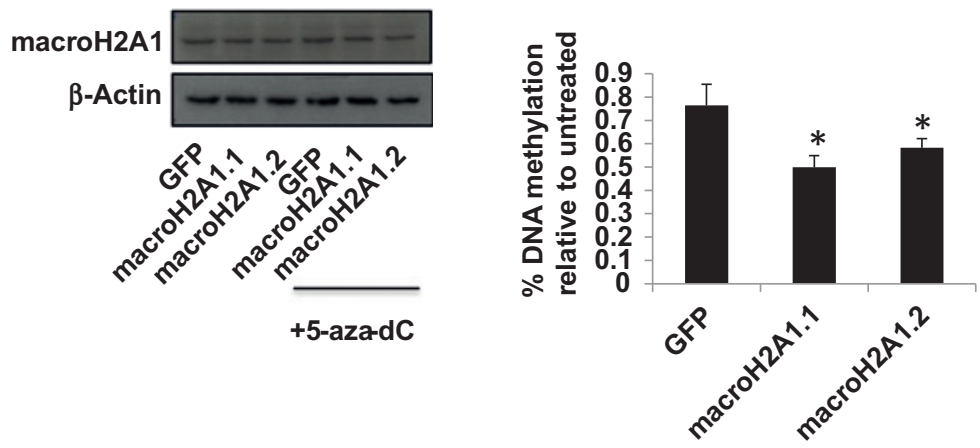

$\mathbf{F}$

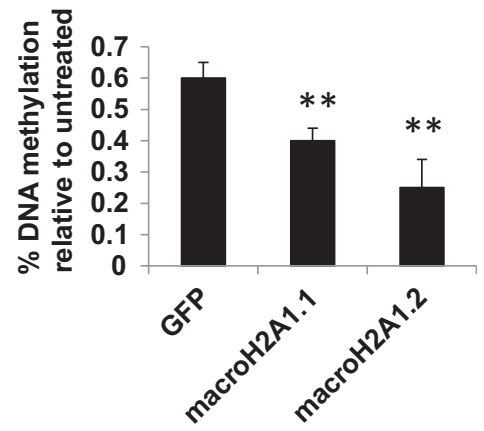

Figure 4.

Treatment with 5-aza-dC of HepG2 and Huh-7 cell lines stable transgenic for GFP, macroH2A1.1, or macroH2A1.2. A and D, chromatin (C) and nuclear (N) fractions isolated from untreated HepG2 (A) or Huh-7 (D) cells transgenic for GFP, macroH2A1.1-GFP, or macroH2A1.2-GFP were processed for immunoblotting with anti-GFP and total anti-macroH2A1 antibodies. Bands corresponding to macroH2A1-GFP chimeras ( $70 \mathrm{KD})$, to the endogenous macroH2A1 ( $40 \mathrm{KD}$ ), and to GFP ( $30 \mathrm{KD})$ are highlighted with red boxes. Histone $\mathrm{H3}$ was used as a marker for the chromatin fraction, whereas negative signal for GAPDH indicated no cytoplasmic contamination. B and E, HepG2 (B) or Huh-7 (E) cells stably overexpressing GFP were treated with $12 \mu$ mol/L 5-aza-dC for 72 hours and processed for immunoblotting with anti-macroH2A1 and macroH2A1.2 antibodies. Bands corresponding to the endogenous macroH2A1.1 and

macroH2A1.2 ( 40 KD) are shown. $\beta$-Actin was used as a loading control. C and F, assessment of global DNA methylation with LUMA assay in HepG2 (C) and Huh-7 (F) cell lines, upon 72-hour treatment with $12 \mu \mathrm{mol} / \mathrm{L} 5$-aza-dC, expressed in a percentage of untreated control. Data were expressed as mean \pm SE of three independent experiments. ${ }^{* *}, P<0.01$.

and phospho-ATF2 (Thr71): confirming the in silico analysis, an increased phosphorylation of p38 MAPK and its downstream effectors was detected in macroH2A1.1 and macroH2A1.2 transgenic HepG2 and Huh-7 cells (Supplementary Fig. S8A and $\mathrm{S} 8 \mathrm{C})$. We then used a selective inhibitor of p38 MAPK activity, SB202190, at a concentration $(10 \mu \mathrm{mol} / \mathrm{L})$ that is cytostatic in both cell lines (Supplementary Fig. S9A and S9B), and previously demonstrated to be effective in inhibiting p38 MAPK in HCC cells (39). Coincubation of HepG2 or Huh-7 cells with SB202190 and 5-aza-dC for 72 hours did not affect hyperphosphorylation of p38 MAPK in macroH2A1 isoform transgenic cells, whereas MSK1 and ATF2 phosphorylation returned to basal levels, as expected (Supplementary Fig. S8A-S8D). We then tested the hypothesis that blockade of p38 kinase or
IL8 could hamper IL8 hyperproduction. Coincubation of HepG2 or Huh-7 cells with SB202190 and 5-aza-dC for 72 hours prevented IL8 hyperproduction in macroH2A1.1-GFP and macroH2A1.2-GFP transgenic cells compared with control cells (Fig. 6B and Supplementary Fig. S5B). In contrast, treatment with potent and selective IL8 receptor (CXCR2) antagonist SB265610 (10 $\mu \mathrm{mol} / \mathrm{L}$; ref. 40) was not effective in blocking the increase of IL8 levels in macroH2A1.1-GFP and macroH2A1.2GFP transgenic cells upon 5-aza-dC treatment (Fig. 6B and Supplementary Fig. S5B). Thus, IL8 binding to its receptor is not apparently involved in IL8 secretion. SB202190 and SB265610 were effective in blocking the macroH2A1.1-GFPand macroH2A1.2-GFP-dependent escape from 5-aza-dCinduced cell senescence as resulted from MTT proliferation 


\section{HepG2}

A

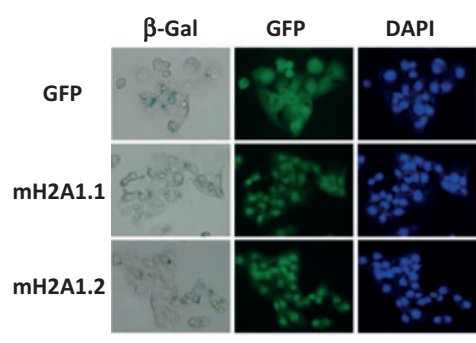

$+5-a z a-d C$
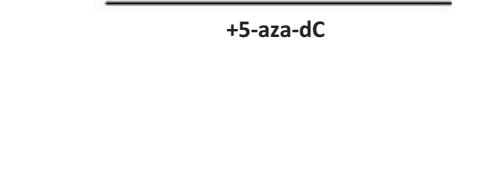

E

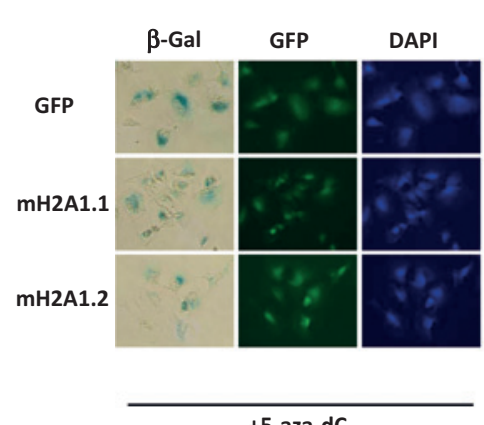

B

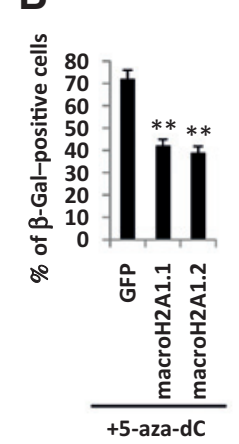

C

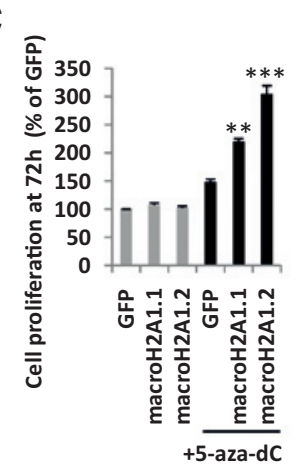

D

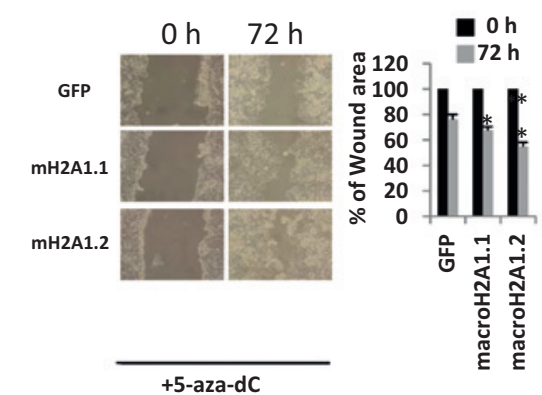

\section{Huh-7}

F

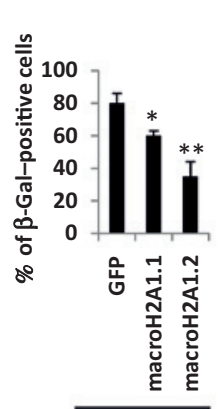

+5 -aza-dC
G

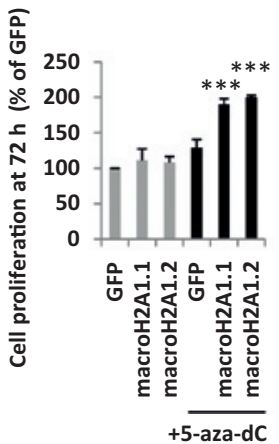

H

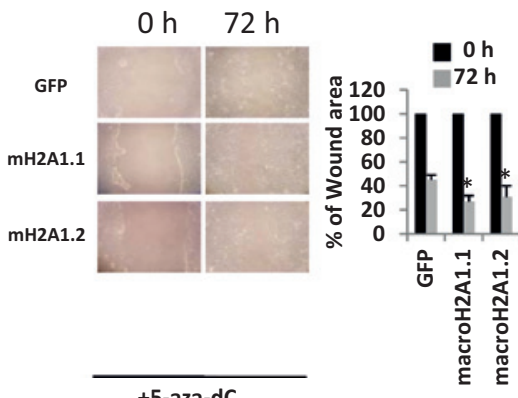

Figure 5.

5-aza-dC-induced senescence and growth inhibition in HepG2 (A-D) and Huh-7 cells (E-H) transgenic for GFP, macroH2A1.1-GFP (macroH2A1.1), or macroH2A1.2-GFP (macroH2A1.2) following treatment with $12 \mu \mathrm{mol} / \mathrm{L} 5$-aza-dC for 72 hours. A and E, $\beta$-gal staining of HepG2 (A) and Huh-7 (E) cells. Nuclei were counterstained with DAPI. Images were taken under bright light for $\beta$-gal and with respective channels for DAPI (blue) and GFP (green). B and F, positive cells for $\beta$-gal staining as in A and $E$ were counted and represented as a proportion of the total. Results are expressed as mean $\pm \mathrm{SE}$ of three experiments. C and G, MTT assay in HepG2 (C) and Huh-7 (G) cells after 72 hours with (black columns) or without (gray columns) 5-aza-dC incubation; percentage growth compared with untreated GFP control cells. D and H, scratch migration assay in HepG2 (D) and Huh-7 (H) cells. Left, representative images taken at 0 and 72 hours after scratching. Right, software-assisted quantification of wounded areas expressed as a percentage of the initial scratch. All data were expressed as mean $\pm \mathrm{SE}$ of three independent experiments. ${ }^{*}, P<0.05$; $^{* *}, P<0.01$; and ${ }^{* * *}, P<0.001$.

assays (Fig. 6C and Supplementary Fig. S5C) and $\beta$-gal staining (Fig. 6D and Supplementary Fig. S5D). In conclusion, macroH2A1 isoforms might confer resistance to drug-induced senescence through a p38 MAPK/IL8 mechanism in HCC cell lines.

Redistribution of macroH2A1 isoform genomic occupancy is largely independent on 5-aza-dC-induced transcriptional regulation

We sought to analyze if changes in chromatin occupancy by macroH2A1.1 and macroH2A1.2 might play a role in 5-aza-dCinduced transcriptional changes. We used ChIP-Seq, using a ChIP grade anti-GFP antibody, to precipitate GFP and macroH2A1-GFP chimeric proteins (Supplementary Fig. S10A). LAMA5 and PTPRN genes were used as positive controls to assess chromatin binding by qRT-PCR, which showed tight macroH2A1 binding to chromatin in macroH2A1.1-GFP and macroH2A1.2-GFP transgenic cells compared with GFP-expressing cells (Supplementary Fig. S10B). Full ChIP-Seq raw data are reported in Supplementary Table S3. 5-aza-dC treatment was associated with differentially
ChIPed genes that were significantly more represented in macroH2A1.1-GFP (449) than in macroH2A1.2-GFP (166)overexpressing cells, with only 60 genes in common (Fig. 7A). AKT3 and STARD8, also known as DLC-3, are shown in Fig. 7B as examples of HCC-related genes displaying differential binding with macroH2A1.1-GFP and macroH2A1.2-GFP, respectively, upon 5-aza-dC treatment. Binding sites were subsequently grouped by gene section, i.e., $3^{\prime}$ or $5^{\prime}$ untranslated region (UTR), coding sequence, intergenic, intron, noncoding, and promoter (Supplementary Fig. S10C). The frequency of occupancy showed that macroH2A1.2 binding was enriched in intergenic and promoter regions, and decreased in introns, $5^{\prime}$ UTR, and noncoding regions, whereas macroH2A1.1 binding was decreased in intergenic regions and increased in introns (Supplementary Fig. S10C). Filtering only binding sites that exhibited $P$ values $<0.05$, MannWhitney $P$ values $<0.1$, and differential scaled changes of at least 0.5 -fold between 5-aza-dC-treated and -untreated cells, and further selecting genes that were differentially expressed $(>1.5$-fold) at the mRNA level between 5-aza-dC-treated 
A

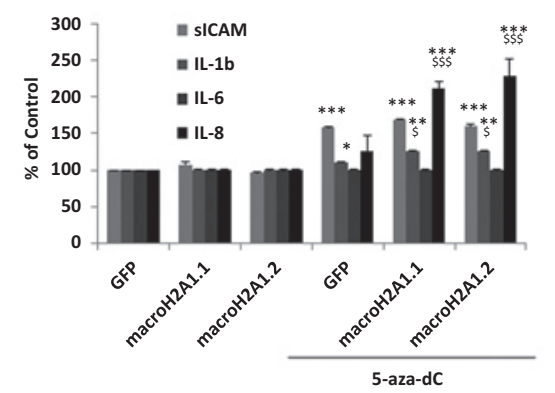

D

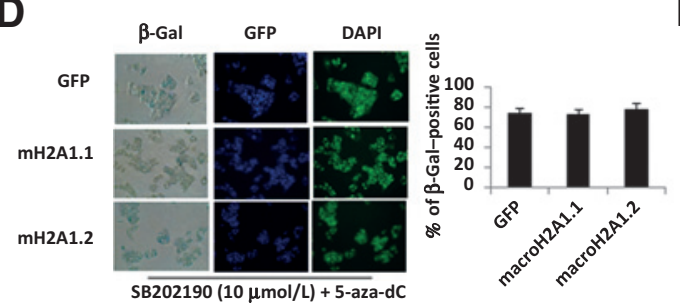

B

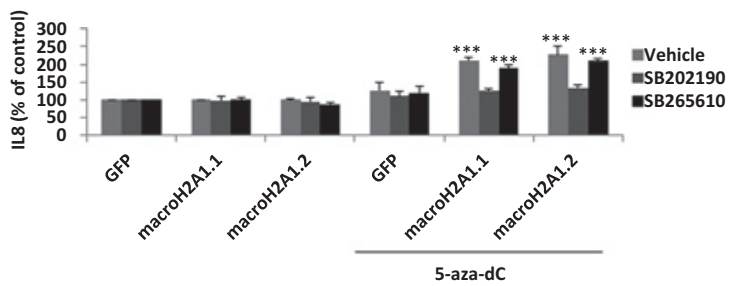

C

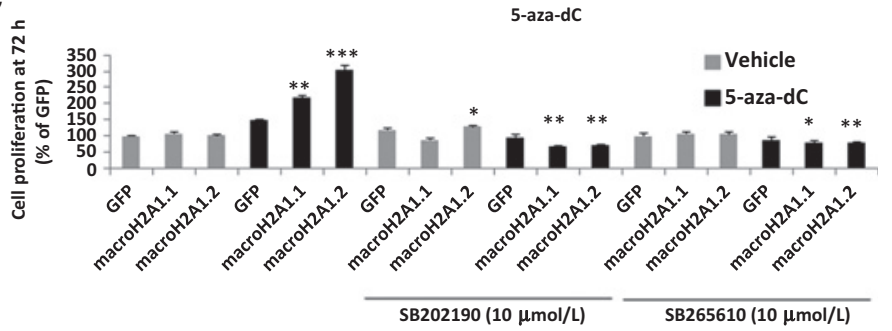

E

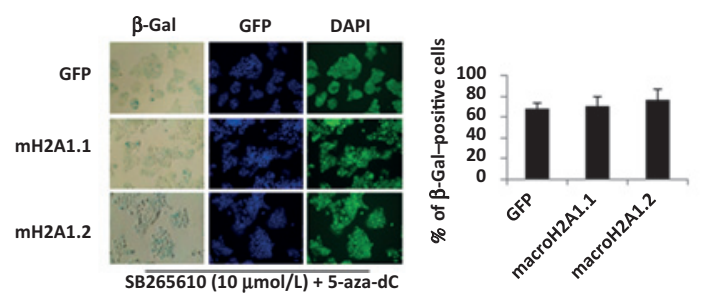

Figure 6.

macroH2A1.1 and macroH2A1.2 counteract 5-aza-dC-induced HepG2 cell senescence in a p38 MAPK/IL8-dependent manner. A, the protein levels of SASP effectors sICAM, IL1b, IL6, and IL8 were assessed in the supernatant of HepG2 cells with or without $12 \mu$ mol/L 5-aza-dC for 72 hours with a customized human MILLIPLEX MAP (multi-analyte panels) Luminex system. B, IL8 levels were assessed in the supernatant of HepG 2 cells with or without $12 \mu$ mol/L 5-aza-dC for 72 hours, and in the presence or absence of selective p38 MAPK inhibitor (SB202190; $10 \mu \mathrm{mol} / \mathrm{L}$ ) or IL8 receptor antagonist (SB265610; $10 \mu \mathrm{mol} / \mathrm{L}$ ), with a MILLIPLEX Luminex System. C, MTT assay in HepG2 cells after 72 hours with (black columns) or without (gray columns) 5-aza-dC incubation; percentage growth compared with untreated GFP control cells in the presence or absence of p38 MAPK inhibitor (SB202190; $10 \mu \mathrm{mol} / \mathrm{L}$ ) or IL8 receptor antagonist (SB265610; $10 \mu \mathrm{mol} / \mathrm{L}$ ). D and E, $\beta$-gal staining of HepG2 cells upon 5 -aza-dC treatment ( $12 \mu \mathrm{mol} / \mathrm{L}$ for 72 hours) in combination with SB202190 (10 $\mu \mathrm{mol} / \mathrm{L}$; $\mathrm{D}$ ) or in combination with SB265610 (10 $\mu \mathrm{mol} / \mathrm{L}$; E). Nuclei were counterstained with DAPI. Images were taken under bright light for $\beta$-gal and with respective channels for DAPI (blue) and GFP (green). All data were expressed as mean \pm SE of three independent experiments. ${ }^{*}, P<0.05 ;{ }^{* *}, P<0.01 ;$ and ${ }^{* * *}, P<0.001$ versus control untreated GFP-expressing cells. $\$, P<0.05$ and $\$ \$ \$, P<0.001$ versus control GFP-expressing cells treated with $12 \mu$ mol/L 5 -aza-dC for 72 hours.

and -untreated cells, we obtained only 28 and 9 genes for macroH2A1.1 and macroH2A1.2, respectively, involved in nutrient metabolism and cancer pathways (Circos plot, Fig. 7C). Our genomic analysis thus demonstrated that 5-aza-dC treatment induces modest changes in macroH2A1.1 and macroH2A1.2 genome occupancy that may not account for its transcriptional effects in HCC cells.

\section{Discussion}

In this study, we describe a new epigenetic synergism between the histone variant macroH2A1 and DNA methylation, controlling the escape of HCC cells from drug-induced senescence. HCC frequently involves genome-wide DNA hypomethylation accompanied by focal promoter hypomethylation and heterochromatinization. Interestingly, global DNA hypomethylation is also a characteristic of most aging tissues, with the exception of the liver (41). Our data in healthy old versus young murine and human liver specimens confirm these findings. Focal DNA methylation signatures have been used as biomarkers of HCC (20), and of healthy liver aging progression (42). The liver is an ideal tissue to study the epigenetic mechanisms that control cell fate toward healthy aging or cancer. We focused on emerging epigenetic players in tumorigenesis, the exon-spliced isoforms of histone variant macroH2A1-macroH2A1.1 and macroH2A1.2 (13)- and their interplay with DNA methylation during aging and HCC. Although macroH2A1.1 acts as a tumor suppressor in many cancer types, the role of macroH2A1.2 is context-dependent (10). MacroH2A1.1 and macroH2A1.2 are dramatically and equally upregulated in HCC (3), reflecting the lack of linearity in macroH2A1 pre-mRNA splicing processes identified in HCC versus adjacent normal liver tissues (43). The whole macroH2A1 gene acts synergistically with 5-aza-dC-induced DNA demethylation to silence the tumor suppressor and senescence marker p16 in fibroblasts (25). We found that an increased expression of macroH2A1 isoforms is associated to senescence marker $\beta$-gal staining in human HCC tissue. Protein levels of macroH2A1 isoforms were also significantly increased without changes in 5-methyl cytosine positivity in the liver of old mice and humans. To study the functional interplay between the macroH2A1 isoforms, HCC-associated DNA hypomethylation, and senescence/ HCC aggressiveness, we established HepG2 and Huh-7 HCC cell lines expressing GFP-, macroH2A1.1-GFP-, and macroH2A1.2GFP transgenes. Consistent with previous reports (21-24), incubation of HepG2 and Huh-7 cells with DNA-demethylating agent 5 -aza-dC induced a senescent-like phenotype, characterized by $\beta$-gal staining, reduced proliferation, and migration. Overexpression of either macroH2A1.1 or macroH2A1.2 counteracted this drug-induced senescent-like phenotype. In addition, macroH2A1 isoform activity induced a decrease in $\beta$-gal staining and an 
A

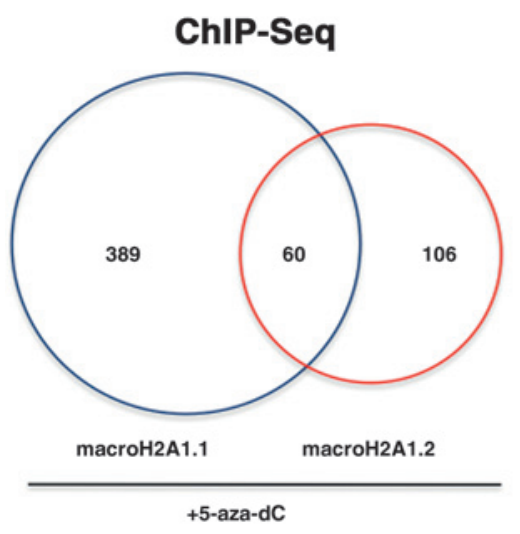

Figure 7.

Representation of commonly and differentially bound genes in ChIP-Seq data. A, Venn diagram of differentially bound genes for macroH2A1.1 + 5-aza$\mathrm{dC}$ versus CTL-GFP +5 -aza-dC and macroH2A1.2 + 5 -aza-dC versus CTL-GFP + 5-aza-dC HepG2 stable cell lines. B, modified Partek Genome Browser view of two representative gene bodies differentially bound (assessed as differential coverage of peaks) by macroH2A1.1 (AKT3) and by macroH2A1.2 (STARD8) following 5-aza-dC treatment. Boxes indicate gene regions of significant differential binding. C, representation of differentially expressed and differentially bound genes and their common pathways in macroH2A1.1overexpressing (left) and macroH2A1.2 transgenic (right) HepG2 cells. The outer circle shows the genomic positions of genes. Heatmaps represent fold change values of RNA-Seq (red) and ChIP-Seq (blue). Blue heatmaps illustrate the binding strength of macroH2A1 isoforms in untreated cells (outer) and following 5-aza-dC treatment (inner) in HepG2 cells. Genes are linked by colored edges based on their involvement in cancer, metabolic, and inflammatory pathways. Genes belonging to the first group are linked in red; genes belonging to the first and second groups are linked in dark green.

C
B
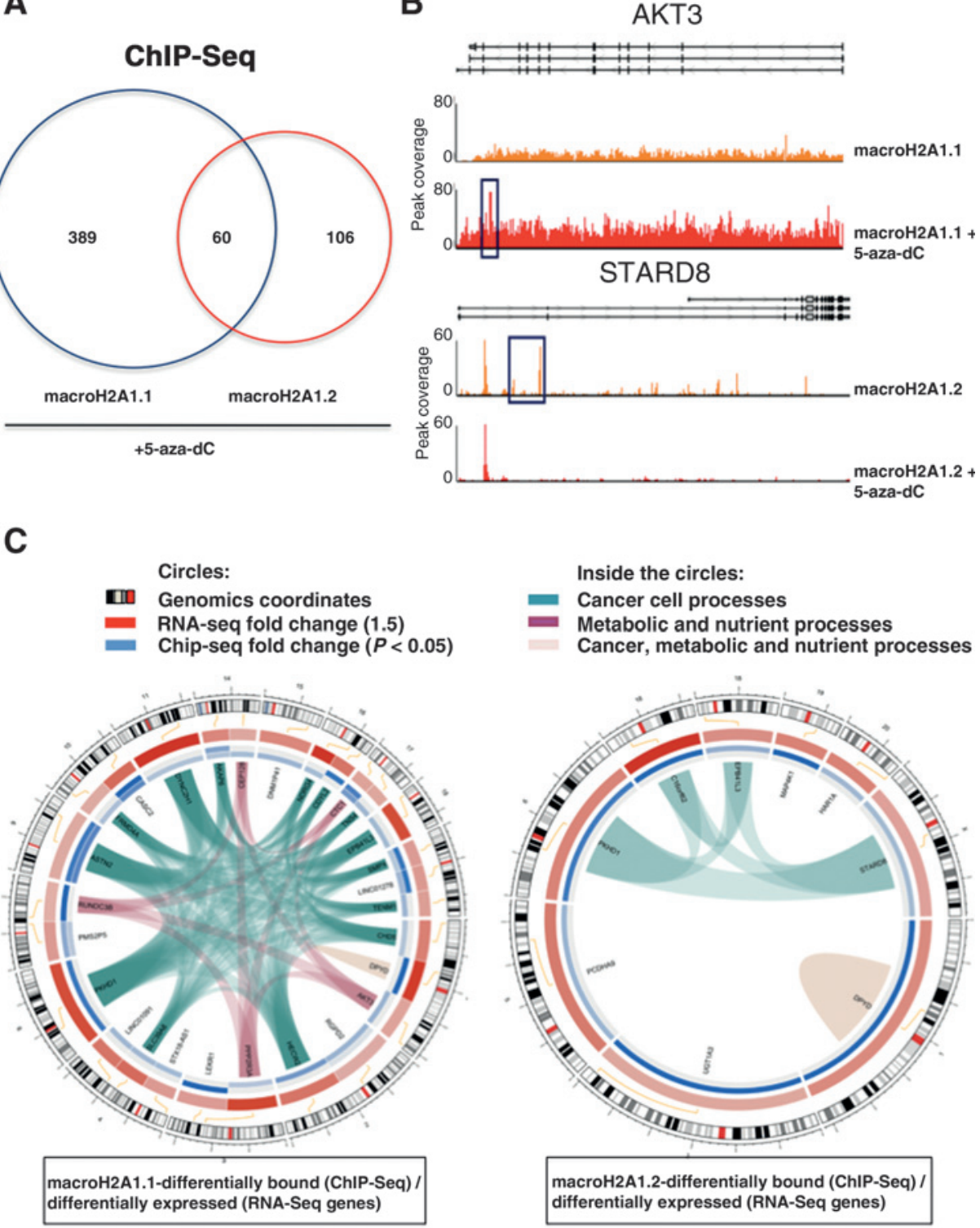

increase in antisenescence gene expression in mouse HCC cells (Hepa1-6), indicating a potential evolutionarly conserved mechanism. Conversely, KD of the macroH2A1 gene slowed cell proliferation and migration compared with control HCC cells. However, KO of macroH2A1 in old mice or KD in 5-aza-dCtreated HepG2 cells had no effects on global DNA methylation and senescence markers. MacroH2A1 function is thus dispensable for age- and drug-induced senescence. The cellular response to DNA hypomethylation is variable. In part, the affected genomic region dictates the cellular response to loss of DNA methylation: hypomethylation of gene regulatory regions, such as promoters, can remove gene repression, whereas hypomethylation of repetitive elements can reduce heterochromatin formation and promote genomic instability. Our data show that transgenic expression of macroH2A1.1 or macroH2A1.2 in HepG2 and in Huh-7 cells paradoxically synergized with 5 -aza-dC to induce a decrement in DNA genome-wide methylation. Further studies are needed to determine which genomic regions are selectively demethylated by macroH2A1 overexpression or by DNAdemethylating drugs in HCC; interestingly, in colorectal cancer cells, 5-aza-dC targets genomic DNA, whereas promoter CpG island methylation remains unchanged (44).

In HCC cells, 5-aza-dC-induced senescence was not accompanied by activation of the classic regulator of senescence p16, p21, and p53. Instead, we report SASP activation, consistent with previous studies (21). Among SASP cytokines/chemokines, IL8 expression displayed a 2 -fold increase in protein levels in cells transgenic for macroH2A1 isoforms upon 5-aza$\mathrm{dC}$ treatment. macroH2A1 was recently shown as a critical component of the feedback loop that maintains SASP in oncogene-induced senescence (45). Our RNA-Seq showed that cells transgenic for macroH2A1 isoforms displayed higher levels of mRNAs involved in oncogenesis than cells depleted of macroH2A1. In detail, IPA indicated a strong enrichment of 
transcripts involved in p38 MAPK activation and subsequent IL8 protein production in HCC cells transgenic for macroH2A1 isoforms. p38 MAPK signaling activation was confirmed by immunoblotting. p38 MAPK and IL8 are implicated in HCC aggressiveness and metastatic potential $(46,47)$. Our findings using highly selective inhibitors of p38 MAPK activity and IL8 receptor activation showed that blockage of either pathway is sufficient to dampen the antisenescence effect of macroH2A1 isoforms. Furthermore, IL8 production by macroH2A1 transgenic HCC cells was dependent on p38 MAPK signaling, a mechanism previously identified for IL8 production in immune cells (48). Campisi's laboratory demonstrated that p38 MAPK activity is necessary and sufficient to regulate SASP, independently of p53 and p21, as in our study, upon a variety of senescence-inducing stresses (49).

Despite having transcriptional and genomic patterns partially overlapping, macroH2A1 isoforms trigger a similar escape from 5aza-dC-induced tumor suppression. Our computational analysis revealed a very limited number of genes with different mRNA levels in 5-aza-dC-treated and -untreated cells that were differentially bound by either one or other of the isoforms. This might suggest indirect oncogenic/SASP transcriptional programs independent of changes in macroH2A1 genomic occupancy. Variations in macroH2A1 transcriptional activities without changes in genome occupancy have been reported in breast cancer cells (8).

In summary, our data indicate that macroH2A1 might be a simple bystander in the liver of the healthy elderly, and it might not be of use to counteract age-associated senescence in the absence of DNA demethylation. Conversely, macroH2A1 could be both a powerful tissue biomarker to stratify HCC patients (3) and a new target to implement SASP- and chemotherapy-based therapeutic opportunities $(24,38,45)$.

\section{Disclosure of Potential Conflicts of Interest}

No potential conflicts of interest were disclosed.

\section{References}

1. Gores GJ. Decade in review-hepatocellular carcinoma: HCC-subtypes, stratification and sorafenib. Nat Rev Gastroenterol Hepatol 2014;11: 645-7.

2. Sheedfar F, Di Biase S, Koonen D, Vinciguerra M. Liver diseases and aging: friends or foes? Aging Cell 2013;12:950-4.

3. Rappa F, Greco A, Podrini C, Cappello F, Foti M, Bourgoin L, et al. Immunopositivity for histone MacroH2A1 isoforms marks steatosis-associated hepatocellular carcinoma. PLoS ONE 2013;8:e54458.

4. Marongiu F, Serra MP, Sini M, Angius F, Laconi E. Clearance of senescent hepatocytes in a neoplastic-prone microenvironment delays the emergence of hepatocellular carcinoma. Aging (Albany NY) 2014;6:26-34.

5. Rai TS, Adams PD. Lessons from senescence: chromatin maintenance in non-proliferating cells. Biochim Biophys Acta 2012;1819:322-31.

6. Kustatscher G, Hothorn M, Pugieux C, Scheffzek K, Ladurner AG. Splicing regulates NAD metabolite binding to histone macroH2A. Nat Struct Mol Biol 2005; 12:624-5

7. Creppe C, Janich P, Cantarino N, Noguera M, Valero V, Musulén E, et al. MacroH2A1 regulates the balance between self-renewal and differentiation commitment in embryonic and adult stem cells. Mol Cell Biol 2012;32: 1442-52.

8. Gamble M, Frizzell KM, Yang C, Krishnakumar R, Kraus WL. The histone variant macroH2A1 marks repressed autosomal chromatin, but protects a subset of its target genes from silencing. Genes Dev 2010;24:21-32.

9. Creppe C, Posavec M, Douet J, Buschbeck M. MacroH2A in stem cells: a story beyond gene repression. Epigenomics 2012;4:221-7.

\section{Authors' Contributions}

Conception and design: G. Rizzo, J.A. Oben, I. Pata, S. Minogue, V. Pazienza, M. Vinciguerra

Development of methodology: M. Borghesan, C. Fusilli, I. Pata, T. Mazza Acquisition of data (provided animals, acquired and managed patients, provided facilities, etc.): M. Borghesan, F. Rappa, C. Panebianco, C. Faulkes, I. Pata, A. Warren, A. Peterson, J.M. Sedivy, J. Douet, M. Buschbeck, M. Vinciguerra

Analysis and interpretation of data (e.g., statistical analysis, biostatistics, computational analysis): M. Borghesan, C. Fusilli, F. Rappa, C. Panebianco, G. Mazzoccoli, J.M. Sedivy, F. Cappello, T. Mazza, V. Pazienza, M. Vinciguerra Writing, review, and/or revision of the manuscript: M. Borghesan, G. Rizzo, J.A. Oben, G. Mazzoccoli, A. Agodi, F. Rezaee, S. Minogue, J.M. Sedivy, J. Douet, M. Buschbeck, T. Mazza, V. Pazienza, M. Vinciguerra

Administrative, technical, or material support (i.e., reporting or organizing data, constructing databases): M. Borghesan, F. Rappa, C. Panebianco, V. Pazienza

Study supervision: M. Borghesan, G. Mazzoccoli, A. Agodi, V. Pazienza, M. Vinciguerra

\section{Acknowledgments}

The authors thank Sara Cannito, Sant'Agata, Lucio Iannone, and the Genome Centre of Bart's and the London School of Medicine and Dentistry (QMUL, London, UK) for technical assistance. They are also grateful to Ron Hogg for proofreading of the article.

\section{Grant Support}

V. Pazienza and M. Vinciguerra are supported by Italian Ministry of Health, Bando GR-2010-2311017. M. Vinciguerra is supported by a My First Associazione Italiana Ricerca sul Cancro (AIRC) Grant-AIRC Grant no. 13419 and by UCL; J. Douet by the Juan de la Cierva Fellowship JCI-2011-10831; and M. Buschbeck by the Ramón y Cajal Fellowship RYC2010-07337 and the grant SAF2012-39749 (MINECO). J.M. Sedivy was supported by grant R37 AG016694 from the NIH.

The costs of publication of this article were defrayed in part by the payment of page charges. This article must therefore be hereby marked advertisement in accordance with 18 U.S.C. Section 1734 solely to indicate this fact.

Received May 19, 2015; revised October 20, 2015; accepted November 2, 2015; published OnlineFirst January 15, 2016.

10. Cantarino N, Douet J, Buschbeck M. MacroH2A-an epigenetic regulator of cancer. Cancer Lett 2013;336:247-52.

11. Posavec M, Timinszky G, Buschbeck M. Macro domains as metabolite sensors on chromatin. Cell Mol Life Sci 2013;70:1509-24.

12. Sheedfar F, Vermeer M, Pazienza V, Villarroya J, Rappa F, Cappello F, et al. Genetic ablation of macrohistone H2A1 leads to increased leanness, glucose tolerance and energy expenditure in mice fed a high-fat diet. Int J Obes (Lond) 2015;39:331-8.

13. Borghesan M, Mazzoccoli G, Sheedfar F, Oben J, Pazienza V, Vinciguerra M. Histone variants and lipid metabolism. Biochem Soc Trans 2014;42: 1409-13.

14. Boulard M, Storck S, Cong R, Pinto R, Delage H, Bouvet P. Histone variant macroH2A1 deletion in mice causes female-specific steatosis. Epigenetics Chromatin 2010;3:8.

15. Changolkar LN, Pehrson JR. macroH2A1 histone variants are depleted on active genes but concentrated on the inactive X chromosome. Mol Cell Biol 2006;26:4410-20.

16. Changolkar LN, Singh G, Cui K, Berletch JB, Zhao K, Disteche CM, et al. Genome-wide distribution of $\mathrm{MacroH} 2 \mathrm{~A} 1$ histone variants in mouse liver chromatin. Mol Cell Biol 2010;30:5473-83.

17. Pazienza V, Borghesan M, Mazza T, Sheedfar F, Panebianco C, Williams R, et al. SIRT1-metabolite binding histone macroH2A1.1 protects hepatocytes against lipid accumulation. Aging (Albany NY) 2014;6:35-47.

18. Podrini C, Koffas A, Chokshi S, Vinciguerra M, Lelliott CJ, White JK, et al. $\mathrm{MacroH} 2 \mathrm{~A} 1$ isoforms are associated with epigenetic markers for activation of lipogenic genes in fat-induced steatosis. FASEB J 2015;29:1676-87. 
19. Kreiling JA, Tamamori-Adachi M, Sexton AN, Jeyapalan JC, MunozNajar U, Peterson AL, et al. Age-associated increase in heterochromatic marks in murine and primate tissues. Aging Cell 2011;10: 292-304.

20. Calvisi DF, Ladu S, Gorden A, Farina M, Lee JS, Conner EA, et al. Mechanistic and prognostic significance of aberrant methylation in the molecular pathogenesis of human hepatocellular carcinoma. J Clin Invest 2007; 117:2713-22.

21. Venturelli S, Berger A, Weiland T, Essmann F, Waibel M, Nuebling T, et al. Differential induction of apoptosis and senescence by the DNA methyltransferase inhibitors 5-azacytidine and 5-aza-2'-deoxycytidine in solid tumor cells. Mol Cancer Ther 2013;12:2226-36.

22. Venturelli S, Berger A, Weiland T, Zimmermann M, Häcker S, Peter C, et al. Dual antitumour effect of 5-azacytidine by inducing a breakdown of resistance-mediating factors and epigenetic modulation. Gut 2011;60: 156-65.

23. Venturelli S, Armeanu S, Pathil A, Hsieh CJ, Weiss TS, Vonthein R, et al. Epigenetic combination therapy as a tumor-selective treatment approach for hepatocellular carcinoma. Cancer 2007;109:2132-41.

24. Jueliger SL, Lyons JF, Azab M, Taverna P. SGI-110, a novel second generation DNA hypomethylating agent, enhances sorafenib activity and alters the methylation signature of HCC cell lines. EORTC-AACRNCI Symposium on Molecular Targets and Cancer Therapeutics Dublin, Ireland; 2012.

25. Barzily-Rokni M, Friedman N, Ron-Bigger S, Isaac S, Michlin D, Eden A. Synergism between DNA methylation and macroH2A1 occupancy in epigenetic silencing of the tumor suppressor gene p16(CDKN2A). Nucleic Acids Res 2010;39:1326-35.

26. Changolkar LN, Costanzi C, Leu NA, Chen D, McLaughlin KJ, Pehrson JR. Developmental changes in histone macroH2A1-mediated gene regulation. Mol Cell Biol 2007;27:2758-64.

27. McKee C, Sigala B, Soeda J, Mouralidarane A, Morgan M, Mazzoccoli G, et al. Amphiregulin activates human hepatic stellate cells and is upregulated in non alcoholic steatohepatitis. Sci Rep 2015; 5:8812.

28. Benegiamo G, Mazzoccoli G, Cappello F, Rappa F, Scibetta N, Oben J, et al. Mutual antagonism between circadian protein period 2 and hepatitis C virus replication in hepatocytes. PLoS ONE 2013;8:e60527.

29. Kleiner DE, Brunt EM, Van Natta M, Behling C, Contos MJ, Cummings OW, et al. Design and validation of a histological scoring system for nonalcoholic fatty liver disease. Hepatology 2005;41:1313-21.

30. Itahana K, Campisi J, Dimri GP. Methods to detect biomarkers of cellular senescence: the senescence-associated beta-galactosidase assay. Methods Mol Biol 2007;371:21-31.

31. Cong R, Das S, Douet J, Wong J, Buschbeck M, Mongelard F, et al. macroH2A1 histone variant represses rDNA transcription. Nucleic Acids Res 2014;42:181-92.

32. D'Aronzo M, Vinciguerra M, Mazza T, Panebianco C, Saracino C, Pereira $\mathrm{SP}$, et al. Fasting cycles potentiate the efficacy of gemcitabine treatment in in vitro and in vivo pancreatic cancer models. Oncotarget 2015; 6:18545-57.

33. Karimi M, Johansson S, Ekstrom TJ. Using LUMA: a Luminometric-based assay for global DNA-methylation. Epigenetics 2006;1:45-8.
34. Ripoli M, Barbano R, Balsamo T, Piccoli C, Brunetti V, Coco M, et al. Hypermethylated levels of E-cadherin promoter in Huh-7 cells expressing the HCV core protein. Virus Res 2011;160:74-81.

35. Wysocka J, Reilly PT, Herr W. Loss of HCF-1-chromatin association precedes temperature-induced growth arrest of tsBN67 cells. Mol Cell Biol 2001;21:3820-9.

36. Bolasco G, Calogero R, Carrara M, Banchaabouchi MA, Bilbao D, Mazzoccoli $\mathrm{G}$, et al. Cardioprotective mIGF-1/SIRT1 signaling induces hypertension, leukocytosis and fear response in mice. Aging (Albany NY) 2012; 4:402-16

37. Benegiamo G, Vinciguerra M, Mazzoccoli G, Piepoli A, Andriulli A, Pazienza V. DNA methyltransferases 1 and 3b expression in Huh-7 cells expressing HCV core protein of different genotypes. Dig Dis Sci 2012;57: 1598-603.

38. Tchkonia T, Zhu Y, van Deursen J, Campisi J, Kirkland JL. Cellular senescence and the senescent secretory phenotype: therapeutic opportunities. J Clin Invest 2013;123:966-72.

39. Feng YM, Feng CW, Chen SY, Hsieh HY, Chen YH, Hsu CD. Cyproheptadine, an antihistaminic drug, inhibits proliferation of hepatocellular carcinoma cells by blocking cell cycle progression through the activation of P38 MAP kinase. BMC Cancer 2015;15:134.

40. Ma S, Cheng Q, Cai Y, Gong H, Wu Y, Yu X, et al. IL-17A produced by gammadelta $\mathrm{T}$ cells promotes tumor growth in hepatocellular carcinoma. Cancer Res 2014;74:1969-82.

41. Pogribny IP, Vanyushin BF. Age-Related Genomic Hypomethylation. In: Tollefsbol TO, editor. Epigenetic of aging. New York: Springer Science+Business Media, LLC 2010; 2010. p. 11-8.

42. Horvath S, Erhart W, Brosch M, Ammerpohl O, von Schönfels W, Ahrens M, et al. Obesity accelerates epigenetic aging of human liver. Proc Natl Acad Sci U S A 2014;111:15538-43.

43. Novikov L, Park JW, Chen H, Klerman H, Jalloh AS, Gamble MJ. QKImediated alternative splicing of the histone variant MacroH2A1 regulates cancer cell proliferation. Mol Cell Biol 2011;31:4244-55.

44. Mossman D, Kim KT, Scott RJ. Demethylation by 5-aza-2' -deoxycytidine in colorectal cancer cells targets genomic DNA whilst promoter CpG island methylation persists. BMC Cancer 2010;10:366.

45. Chen H, Ruiz PD, McKimpson WM, Novikov L, Kitsis RN, Gamble MJ. MacroH2A1 and ATM play opposing roles in paracrine senescence and the senescence-associated secretory phenotype. Mol Cell 2015; 59:719-31.

46. Huang W, Chen Z, Zhang L, Tian D, Wang D, Fan D, et al. Interleukin-8 induces expression of FOXC1 to promote transactivation of CXCR1 and CCL2 in hepatocellular carcinoma cell lines and formation of metastases in mice. Gastroenterology 2015;149:1053-67 e14.

47. Hsieh YH, Wu TT, Huang CY, Hsieh YS, Hwang JM, Liu JY. p38 mitogenactivated protein kinase pathway is involved in protein kinase Calpharegulated invasion in human hepatocellular carcinoma cells. Cancer Res 2007;67:4320-7.

48. Hoffmann E, Dittrich-Breiholz O, Holtmann H, Kracht M. Multiple control of interleukin-8 gene expression. J Leukoc Biol 2002;72:847-55.

49. Freund A, Patil CK, Campisi J. p38MAPK is a novel DNA damage responseindependent regulator of the senescence-associated secretory phenotype. EMBO J 2011;30:1536-48. 


\section{Cancer Research}

\section{DNA Hypomethylation and Histone Variant macroH2A1 Synergistically Attenuate Chemotherapy-Induced Senescence to Promote Hepatocellular Carcinoma Progression}

Michela Borghesan, Caterina Fusilli, Francesca Rappa, et al.

Cancer Res Published OnlineFirst January 15, 2016.

Updated version Access the most recent version of this article at: doi:10.1158/0008-5472.CAN-15-1336

E-mail alerts Sign up to receive free email-alerts related to this article or journal.

Reprints and To order reprints of this article or to subscribe to the journal, contact the AACR Publications Subscriptions Department at pubs@aacr.org.

Permissions To request permission to re-use all or part of this article, contact the AACR Publications Department at permissions@aacr.org. 